Consensus Statement

\title{
Terminology for osteochondral lesions of the ankle: proceedings of the International Consensus Meeting on Cartilage Repair of the Ankle
}

\section{Christopher D. Murawski ${ }^{\text {a, }}$, M. Shazil Jamal ${ }^{\text {ae }}$, Eoghan T. Hurley ${ }^{\mathrm{d}}$, Roberto Buda ${ }^{\mathrm{u}}$,}

Kenneth Hunt ${ }^{\text {ac }}$, Graham McCollum ${ }^{\text {ah }}$, Jochen Paul ${ }^{\text {aj }}$, Francesca Vannini ${ }^{\text {ao }}$, Markus Walther ${ }^{\text {aq }}$,

Youichi Yasui ${ }^{\text {ar }}$, Zakariya Ali ${ }^{\mathrm{c}}$, J Nienke Altink ${ }^{\mathrm{b}}$, Jorge Batista ${ }^{\mathrm{e}}$, Steve Bayer ${ }^{\mathrm{a}}$,

Gregory C. Berlet ${ }^{\mathrm{f}}$, James D.F. Calder ${ }^{\mathrm{v}}$, Jari Dahmen ${ }^{\mathrm{b}}$, Martin S. Davey ${ }^{\mathrm{c}}$, Pieter D'Hooghe ${ }^{\mathrm{w}}$,

Christopher W. DiGiovanni ${ }^{g}$, Richard D. Ferkel ${ }^{\mathrm{h}}$, Arianna L. Gianakos ${ }^{\mathrm{g}}$, Eric Giza ${ }^{\mathrm{x}}$,

Mark Glazebrook $^{\mathrm{y}}$, Laszlo Hangody ${ }^{\mathrm{z}}$, Daniel Haverkamp ${ }^{\mathrm{aa}}$, Beat Hintermann ${ }^{\mathrm{ab}}$, Yinghui Hua ${ }^{\mathrm{i}}$,

Daire J. Hurley ${ }^{\text {ad }}$, Jón Karlsson ${ }^{\text {af }}$, Stephen Kearns ${ }^{j}$, John G. Kennedy ${ }^{\text {ag }}$, Gino M.M.J. Kerkhoffs ${ }^{b}$,

Kaj Lambers $^{\mathrm{b}}$, Jin Woo Lee ${ }^{\mathrm{k}}$, Nathaniel P. Mercer ${ }^{\mathrm{ag}}$, Conor Mulvin ${ }^{\mathrm{c}}$, James A. Nunley ${ }^{\mathrm{ai}}$,

Christopher Pearce $^{1}$, Helder Pereira ${ }^{\mathrm{m}}$, Marcelo Prado ${ }^{\mathrm{n}}$, Steven M. Raikin ${ }^{\mathrm{o}}$, Ian Savage-Elliott ${ }^{\text {ak }}$,

Lew C. Schon ${ }^{\text {p, ag }}$, Yoshiharu Shimozono ${ }^{\text {al }}$, James W. Stone ${ }^{\text {q }}$, Sjoerd A.S. Stufkens ${ }^{\text {b }}$,

Martin Sullivan $^{r}$, Masato Takao ${ }^{\mathrm{s}}$, Hajo Thermann ${ }^{\mathrm{am}}$, David Thordarson ${ }^{\text {an }}$, James Toale ${ }^{\mathrm{c}}$,

Victor Valderrabano ${ }^{\mathrm{t}}$, Christiaan J.A. van Bergen ${ }^{\mathrm{ap}}$, C. Niek van Dijk ${ }^{\mathrm{b}}$, Raymond J. Walls ${ }^{\mathrm{ag}}$,

Alastair S. Younger ${ }^{\text {as }}$, MaCalus V. Hogan ${ }^{a}$

${ }^{\text {a }}$ University of Pittsburgh Medical Center, Department of Orthopaedic Surgery, Pittsburgh, PA, USA

${ }^{\mathrm{b}}$ Amsterdam UMC, University of Amsterdam, Department of Orthopaedic Surgery, Amsterdam Movement Sciences, Amsterdam, Netherlands

${ }^{\mathrm{c}}$ Royal College of Surgeons Ireland, Dublin, Ireland

${ }^{\mathrm{d}}$ Sports Surgery Clinic, Dublin, Ireland

${ }^{\mathrm{e}}$ Department of Arthroscopy, Centro Artroscopico Jorge Batista SA, Buenos Aires, Argentina

${ }^{\mathrm{f}}$ Orthopedic Foot and Ankle Center, Worthington, OH, USA

${ }^{g}$ Department of Orthopaedic Surgery, Massachusetts General Hospital, Boston, MA, USA

${ }^{\mathrm{h}}$ Southern California Orthopedic Institute, Los Angeles, CA, USA

${ }^{\mathrm{i}}$ Department of Sports Medicine, Huashan Hospital, Fudan University, Shanghai, China

${ }^{\mathrm{j}}$ National University of Ireland Galway, Galway, Ireland

${ }^{\mathrm{k}}$ Department of Orthopaedic Surgery, Yonsei University College of Medicine, Seoul, South Korea

${ }^{1}$ National University Heath System, Division of Foot and Ankle Surgery, Singapore

${ }^{\mathrm{m}}$ Knee and Ankle Unit of Orthopedic Department of Póvoa de Varzim - Vila Do Conde, Porto, Portugal

${ }^{\mathrm{n}}$ Orthopedics Department, Hospital Israelita Albert Einstein, São Paulo, Brazil

${ }^{\circ}$ Rothman Institute, Department of Orthopaedic Surgery, Thomas Jefferson University Hospital, Philadelphia, PA, USA

${ }^{\mathrm{p}}$ Institute for Foot and Ankle Reconstruction, Mercy Medical Center, Baltimore, MD, USA

${ }^{\mathrm{q}}$ The Orthopedic Institute of Wisconsin, Franklin, WI, USA

${ }^{\mathrm{r}}$ St Vincent's Clinic, Sydney, Australia

${ }^{s}$ Clinical and Research Institute for Foot and Ankle Surgery, Jujo Hospital, Chiba, Japan

${ }^{\mathrm{t}}$ Swiss Ortho Center, Basel, Switzerland

" Clinic of Orthopaedics and Traumatology, SS. Annunziata Hospital, University of Chieti-Pescara, Italy

${ }^{\mathrm{v}}$ Fortius Clinic, London, United Kingdom

${ }^{\mathrm{w}}$ Department of Orthopaedic Surgery, Aspetar Orthopaedic and Sports Medicine Hospital, Doha, Qatar

${ }^{\mathrm{x}}$ Department of Orthopaedics, University of California, Davis, Sacramento, CA, USA

${ }^{y}$ Dalhousie University \& Queen Elizabeth II Health Sciences Centre, Halifax, Nova Scotia, Canada

${ }^{\mathrm{z}}$ Semmelweis University, Department of Traumatology, Uzsoki Hospital, Department of Orthopedics, Budapest, Hungary

${ }^{\text {aa }}$ Xpert Clinics Orthopedics, Amsterdam, the Netherlands

${ }^{\mathrm{ab}}$ Clinic of Orthopaedic Surgery, Kantonsspital Baselland, Liestal, Switzerland

${ }^{\text {ac }}$ Department of Orthopaedic Surgery, University of Colorado School of Medicine, Aurora, CO, USA

${ }^{\text {ad }}$ Mater Misericordiae University Hospital, Dublin, Ireland

ae Royal London Hospital, London, United Kingdom

\footnotetext{
* Corresponding author. Department of Orthopaedic Surgery, 3471 Fifth Avenue, Suite 911, Pittsburgh, PA 15213 , USA. Tel.: +1 412 605 3262. E-mail address: cdmurawski@gmail.com (C.D. Murawski).
} 
${ }^{\text {af }}$ Department of Orthopaedics, Sahlgrenska University Hospital, Sahlgrenska Academy, Gothenburg University, Göteborg, Sweden

${ }^{\text {ag }}$ NYU Langone Health, New York, NY, USA

${ }^{\text {ah }}$ Department Orthopaedics, University of Cape Town, Cape Town, South Africa

ai Department of Orthopaedic Surgery, Duke University, Durham, NC, USA

aj Rennbahnklinik, Muttenz, Basel, Switzerland

${ }^{\mathrm{ak}}$ Department of Orthopedic Surgery, Tulane University School of Medicine, New Orleans, LA, USA

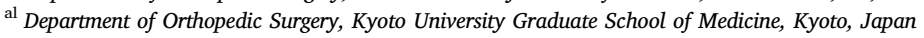

${ }^{\text {am }}$ ATOS Clinic, Heidelberg, Germany

${ }^{\text {an }}$ Department of Orthopaedic Surgery, Cedars Sinai Medical Center, Los Angeles, CA, USA

${ }^{\text {ao }}$ Istituti Ortopedici Rizzoli, Bologna University, Bologna, Italy

${ }^{\text {ap }}$ Department of Orthopaedic Surgery, Amphia, Breda, Netherlands

${ }^{\text {aq }}$ Schoen Klinik Muenchen Harlaching, Academic Hospital of Ludwig-Maximilians-University Munich, Munich, Germany

${ }^{\text {ar }}$ Department of Orthopaedic Surgery, Teikyo University School of Medicine, Tokyo, Japan

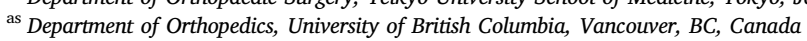

\section{A R T I C L E I N F O}

Keywords:

Ankle

Cartilage

Osteochondral lesion

Talus

\begin{abstract}
A B S T R A C T
Background: The evidence supporting best practice guidelines in the field of cartilage repair of the ankle is based on both low quality and low levels of evidence. Therefore, an international consensus group of experts was convened to collaboratively advance toward consensus opinions based on the best available evidence on key topics within cartilage repair of the ankle. The purpose of this article is to report the consensus statements on "terminology for osteochondral lesions of the ankle" developed at the 2019 International Consensus Meeting on Cartilage Repair of the Ankle.

Methods: Forty-three international experts in cartilage repair of the ankle representing 20 countries were convened and participated in a process based on the Delphi method of achieving consensus. Questions and statements were drafted within four working groups focusing on specific topics within cartilage repair of the ankle, after which a comprehensive literature review was performed, and the available evidence for each statement was graded. Discussion and debate occurred in cases where statements were not agreed on in unanimous fashion within the working groups. A final vote was then held, and the strength of consensus was characterised as follows: consensus, 51\%-74\%; strong consensus, 75\%-99\%; unanimous, $100 \%$.

Results: A total of 11 statements on terminology and classification reached consensus during the 2019 International Consensus Meeting on Cartilage Repair of the Ankle. Definitions are provided for osseous, chondral and osteochondral lesions, as well as bone marrow stimulation and injury chronicity, among others. An osteochondral lesion of the talus can be abbreviated as OLT.

Conclusions: This international consensus derived from leaders in the field will assist clinicians with the appropriate terminology for osteochondral lesions of the ankle.
\end{abstract}

\section{Introduction}

The descriptions of cartilage injuries in the ankle have evolved over the past several centuries. In fact, one of the earliest descriptions of these injuries can be attributed to Monro [1], who in 1737 described the removal of osteocartilaginous loose bodies from the ankle that were believed to be of traumatic origin. Since then, umbrella terms have been used to describe injuries and anomalies of the talus and adjacent articular cartilage. Historically, in 1888, Konig [2] used the term osteochondritis dissecans to loose bodies in the knee, and Kappis [3] subsequently applied the same term to describe similar lesions in the ankle in 1922 [2, 3]. Several subsequent terms and synonyms have been used throughout the 20th century [4]. Given the numerous terms and definitions that have been used, no consensus on the terminology to describe cartilage lesions of the ankle has been developed.

As a whole, the current body of evidence in the field of ankle cartilage repair is based on both low-level and low-quality of evidence [5]. The vast majority of studies are of level IV evidence and consist of short-term follow-up time periods. Therefore, an international, multidisciplinary group of experts was assembled to develop expert and evidence-based consensus statements to assist clinicians in following best practice guidelines. The purpose of this article is to report the results of the working group on "terminology" that were developed at the 2019 International Consensus Meeting on Cartilage Repair of the Ankle.

\section{Methods}

Forty-three national and international multidisciplinary experts in cartilage repair of the ankle were convened to participate in a 1-year consensus building effort, which culminated with the International
Consensus Meeting on Cartilage Repair of the Ankle on March 29-30, 2019 at the Royal College of Surgeons in Dublin, Ireland. This consensus effort followed the previous 2017 initiative in Pittsburgh, the results of which have been published previously [6-16]. Delegates from 20 countries encompassing six continents were represented in the initiative. Experts were assigned to one of four working groups defined by specific subtopics within cartilage repair of the ankle, including "Terminology for Osteochondral Lesions of the Ankle."

Each working group was assigned two liaisons who served as the primary point of contact and dealt with communication and the distribution of surveys. In addition, liaisons were then responsible for writing the surveys, performing data analysis and carrying out literature reviews. To reduce the potential for bias in the data analysis and/or literature review, liaisons did not submit answers to the questionnaires or partake in the voting process. One individual (CDM) maintained oversight of the consensus process to ensure consistency across the working groups.

A modification of the Delphi format described by Linstone and Turoff was used to pursue agreement among the experts on each question. With a few modifications, the specific process that we used for this effort has been described by our group previously [17]. Blinded, electronic surveys were distributed, through which no identifying information was collected. Initially, participants within each working group were asked to submit a list of questions which they felt would contribute meaningfully to areas of current controversy within the specific working group. These lists were then curated for clarity and the removal of duplicates, and a total of 11 questions were formalised on terminology, at which point the process to answer the questions and develop consensus statements was initiated.

Participants were then asked to provide their answers to each question in an open-ended format. These initial open-ended answers then facilitated the development of a more structured questionnaire, with 
emphasis on identifying areas of common ground and resolving aspects of disagreement. Using the results of the third questionnaire, preliminary consensus statements were developed, and a comprehensive literature review was performed to identify, where possible, whether each statement was supported or refuted by the best available evidence. In addition, the available evidence for each statement was graded (Table 1). After the literature review, each group had the opportunity to amend the preliminary statements. Thereafter, a fourth questionnaire requested that each participant "agree" or "disagree" with each preliminary statement. For questions that were agreed on unanimously within the working group, these were progressed to a final vote among all 43 members of the consensus group. For questions that did not achieve unanimous agreement within the working group, these were advanced to an in-person discussion among all participants at the meeting in Dublin.

Seven questions in this working group were not agreed on unanimously and were therefore advanced to the full group, with in-person discussion based on a standardised format. Briefly, each question and proposed answer was presented to the group, after which an opportunity for amendments was provided. Each proposed amendment required two additional participants to second and third the motion. If the amendment was successfully furthered, an opportunity for rebuttal was provided, followed by a vote of agreement or disagreement. In cases where 66\% (2/ 3 's supermajority) or greater of the total votes received were in favour of the proposed amendment, the statement was amended accordingly. This process was repeated for any further amendments that were desired, after which a final vote on the entirety of the statement was undertaken. Voting was conducted using electronic keypads. Similar to the survey data that were collected, all votes were anonymous and of equal weight among participants.

After the final votes for each question occurred, the degree of agreement was expressed using a percentage rounded to the nearest whole number. Consensus was defined as $51-74 \%$, whereas strong consensus was defined as $75-99 \%$, and unanimous was indicated by receiving $100 \%$ of the votes in favour of a proposed statement.

\section{Results}

Of the 11 total questions and consensus statements in this group, four achieved unanimous consensus, and seven achieved strong consensus.

Question: What is the definition of an isolated osseous lesion and what is the appropriate terminology and acronym?

Answer: The definition of an isolated osseous lesion is a bony/subchondral defect absent of any cartilaginous/chondral injury. The appropriate terminology is a subchondral bone lesion, which can be abbreviated as SBL. It should be noted that isolated osseous lesions are rare; chondral lesions often cannot be reliably ruled out (even on MRI) and may require arthroscopy to definitively do so.

Vote: Agree: $98 \%$; disagree: $2 \%$ (strong consensus).

Grade of evidence: $\mathrm{E}$.

Question: What is the definition of an isolated chondral lesion and what is the appropriate terminology and acronym?

Answer: The definition of an isolated chondral lesion is an isolated cartilaginous defect not involving the subchondral bone. The appropriate terminology is a chondral lesion. There was no consensus regarding an appropriate acronym.

Table 1

Grades of evidence.

\begin{tabular}{ll}
\hline A1 & Multiple (two or more) level I randomized controlled trials(RCTs) \\
& with similar findings or a meta-analysis \\
A2 & A single-level I RCT \\
B1 & Prospective cohort study \\
B2 & Any comparison group that is not level I (e.g., case control) \\
C & Case series \\
D & Case report \\
E & Expert opinion/basic science \\
\hline
\end{tabular}

Vote: Agree: 98\%; disagree: $2 \%$ (strong consensus).

Grade of evidence: E.

Question: What is the definition of a combined osseous and chondral lesion and what is the appropriate terminology and acronym?

Answer: The definition of a combined osseous and chondral lesion is a lesion of both the cartilage and subchondral bone. The appropriate terminology is an osteochondral lesion of the talus, which can be abbreviated as OLT.

Vote: Agree: 100\%; disagree: 0\% (Unanimous).

Grade of evidence: E.

Question: What is the appropriate terminology for bone marrow stimulation procedures (e.g. microfracture, drilling)?

Answer: Bone marrow stimulation was an appropriate umbrella term, which can then be further subdivided into microfracture and drilling depending on the specific procedure that is performed. This can be further described based on the particular type of instrumentation used (i.e. the size of drill or awl), as well as whether curettage and/or chondroplasty were performed.

Vote: Agree: 100\%; disagree: 0\% (Unanimous).

Grade of evidence: $\mathrm{E}$.

Question: What is the appropriate terminology and acronym(s) for osteochondral grafts?

Answer: The appropriate terminology for osteochondral grafts is autologous osteochondral transplant (AuOT) or allograft osteochondral transplant (AlOT).

Vote: Agree: 100\%; disagree: 0\% (unanimous).

Grade of evidence: E.

Question: How should subchondral oedema be defined?

Answer: Subchondral oedema is defined as an increase in fluid content of subchondral bone, characterised by a change in subchondral bone signal on MRI.

Vote: Agree: 100\%; disagree: 0\% (unanimous).

Grade of evidence: $\mathrm{E}$.

Question: How can the degree of subchondral oedema be assessed? Answer: The degree of subchondral oedema can be assessed by MRI scan in three planes and its evolution by sequential MRI scans.

Vote: Agree: 98\%; disagree: $2 \%$ (strong consensus).

Grade of evidence: E.

Question: How should a subchondral cyst(s) be defined?

Answer: The definition of a subchondral cyst(s) should include a lesion deep to the chondral surface and adjacent to the subchondral plate of the lesion. A subchondral cyst can be further characterised by the following factors: 1) consistency (e.g. loculated vs. multiloculated); 2) presence or absence of communication with the joint; 3) depth/size; 4) presence or absence of a bone wall surrounding the cyst(s) and 5) location of the cyst(s).

Vote: Agree: $98 \%$; disagree: $2 \%$ (strong consensus).

Grade of evidence: $E$.

Question: With respect to lesion size, what are the appropriate definitions for "area," "diameter" and "depth"?

Answer: "Area" defines the maximal size of the lesion based on the maximal width and diameter of the lesion. Maximal width and diameter can be defined as the medial to lateral and anterior to posterior dimensions, respectively. The "diameter" of a lesion is defined as the maximal width of the lesion at its surface. The "depth" of a lesion is defined as the largest distance from the articular surface to the bottom of the lesion. Measurements should be expressed in millimetres (mm).

Vote: Agree: 95\%; disagree: 5\% (strong consensus).

Grade of evidence: E.

Question: How can subchondral oedema and/or cystic change be classified and is it meaningful for therapeutic or prognostic purposes? 
Answer: The following factors are important in classifying oedema: 1) size; 2) depth; 3) location; 4) total volume and 5) presence or absence of communication with the joint. Although the therapeutic/prognostic value of subchondral oedema is unknown, there is therapeutic/prognostic value in subchondral cystic change.

Vote: Agree: 95\%; disagree: 5\% (strong consensus).

Grade of evidence: E.

Question: Which time parameters define "acute," "subacute" and "chronic" for cartilage injuries of the ankle?

Answer: "Acute" can be defined as $<1$ month, "subacute" as $>1$ month and $<6$ months and chronic as $>6$ months from the onset of symptoms.

Vote: Agree: 86\%; disagree: 14\% (Strong Consensus).

Grade of Evidence: E.

\section{Discussion}

A total of 11 statements on "terminology" reached consensus during the 2019 International Consensus Meeting on Cartilage Repair of the Ankle. Of the 11 consensus statements, four achieved unanimous support, and seven reach reached strong consensus (greater than $75 \%$ agreement). All statements reached at least $86 \%$ agreement. The consensus group also sought to devise a comprehensive classification system for OLT, but this was ultimately deemed not possible in the timespan of our process; this specific topic will be advanced to a special working group in future iterations of the meeting to study this important topic.

Descriptions of cartilaginous or osteochondral injuries in the ankle can be traced back centuries, with one of the earliest known descriptions by Monro in 1737 [1]. Since then, numerous terms have been used to describe these injuries, including but not limited to osteochondral defect, osteochondritis dissecans, joint mice, transchondral fractures, osteochondral lesions and so on [4]. Moreover, several acronyms have been used, including OCL, OLT and OCD, with the latter being often used interchangeably to describe both osteochondral defects and osteochondritis dissecans lesions in multiple joints [4]. It is for these reasons that the purpose of this particular working group within the consensus initiative was to set forth guidelines for the most appropriate terminology for use in the ankle.

The consensus among the group was that the terminology for ankle lesions be based on the specific clinic pathology, namely, whether the lesion involves the bone, cartilage or both. In this regard, subchondral bone lesion (SBL), chondral and osteochondral lesion were the preferred clinical terms, respectively. True osseous or isolated chondral lesions are rare and typically require a combination of imaging and/or arthroscopy to make a definitive diagnosis. In cases where an osteochondral lesion occurs on the talar side of the joint, osteochondral lesion of the talus or OLT is the preferred terminology.

Lesion size is recognised as an important prognostic indicator for surgery in the talus, particularly in the setting of bone marrow stimulation. Despite this, studies to date have reported lesion size using a variety of methods, including diameter, area, volume, depth or a combination of thereof [18]. Even so, it has been previously described by Hannon et al. [19] that some description of lesion size was only reported in $46 \%$ of studies through June 2011. An improved consensus on the definition of lesion size measurements is therefore beneficial, and the group defined area, diameter and depth for use in future clinical practice and studies. In fact, we therefore suggest that all of these parameters in combination with an associated morphological representation of the lesion be reported by authors wherever possible so that, moving forward, the pooling of data and meta-analysis may be possible.

Subchondral oedema and subchondral cystic change are distinct clinical entities and may be important prognostic factors in those with an OLT. Depending on the clinical scenario, the presence of subchondral oedema is unclear as to the meaning but may be present postoperatively for up to two years [16]. Although no consensus was attained with respect to the prognostic or therapeutic effect(s) of subchondral edema, Shimozono et al. [20] found that patients with the presence of subchondral bone marrow oedema at midterm follow-up after bone marrow stimulation for OLT had worse clinical outcomes than those without. In addition, the degree of subchondral bone marrow oedema at midterm follow-up was correlated with worse clinical outcomes. However, at short-term follow-up, there were no significant differences in clinical outcomes based on both the presence and degree of bone marrow oedema, and no correlation was found between clinical outcomes and the degree of bone marrow oedema.

Similarly, subchondral cysts are commonly reported in up to half of OLT cases managed surgically on follow-up imaging. Shimozono et al. [21] reported that the presence of subchondral cysts after autolougous osteochondral transplantation for OLT tends to occur at the graft-host interface, often indicating potential graft failure. The consensus group did agree that there may be prognostic or therapeutic value in understanding the degree of cystic change and that these factors should be classified during evaluation. Savage-Elliott et al. [22] found that although $65 \%$ of patients who underwent autologous osteochondral transplantation had evidence of cystic change on MRI at a mean of 15 months after surgery, an impact on clinical outcomes was not detected at short-term follow-up. Further work by the consensus group and leaders in the field will be required to assess if and/or how oedema and cystic change may affect outcomes in the long-term in these patient populations.

This consensus effort is not without limitations. By definition, consensus statements are considered level $\mathrm{V}$ data and represent a blend of expert opinion and the best available evidence [23]. Nonetheless, the lack of high-quality clinical evidence to date in this field encouraged us to seek alternative methods for developing best practice guidelines in conjunction with leaders in the field. Further high-level studies should be required to substantiate the statements that have been developed as part of this initiative. The consensus will be updated in the event that further evidence for or against a current statement becomes available.

This international consensus derived from leaders in the field will assist clinicians with the appropriate terminology for osteochondral lesions of the ankle.

\section{Competing interests}

None declared.

\section{References}

[1] Monro A. Part of the cartilage of the joint separated and ossified. In: Medical essays and observations. 2nd ed. Edinburgh: Ruddimans; 1737. p. 305

[2] Konig F. Ueber freie korper in den gelenken. Dtsch Z Chir 1887;27:90-109.

[3] Kappis M. Weitese Beitrage zur Traumatisch-mechanischen-Entstehung der "Spontanen" Knospelablosungen. Dtsch Z Chir 1922;171:13-29.

[4] O'Loughlin PF, Heyworth BE, Kennedy JG. Current concepts in the diagnosis and treatment of osteochondral lesions of the ankle. Am J Sports Med 2010;38(2): 392-404. https://doi.org/10.1177/0363546509336336.

[5] Pinski JM, Boakye LA, Murawski CD, Hannon CP, Ross KA, Kennedy JG. Low level of evidence and methodologic quality of clinical outcome studies on cartilage repair of the ankle. Arthroscopy 2016;32(1):214-222 e1. https://doi.org/10.1016/ j.arthro.2015.06.050.

[6] D'Hooghe P, Murawski CD, Boakye LAT, et al. Rehabilitation and return to sports: proceedings of the international consensus meeting on cartilage repair of the ankle. Foot Ankle Int 2018;39(1_suppl):61S-7S. https://doi.org/10.1177/ 1071100718781862.

[7] Dombrowski ME, Yasui Y, Murawski CD, et al. Conservative management and biological treatment strategies: proceedings of the international consensus meeting on cartilage repair of the ankle. Foot Ankle Int 2018;39(1_suppl):9S-15S. https:// doi.org/10.1177/1071100718779390.

[8] Hannon CP, Bayer S, Murawski CD, et al. Debridement, curettage, and bone marrow stimulation: proceedings of the international consensus meeting on cartilage repair of the ankle. Foot Ankle Int 2018;39(1_suppl):16S-22S. https://doi.org/10.1177/ 1071100718779392.

[9] Hurley ET, Murawski CD, Paul J, et al. Osteochondral autograft: proceedings of the international consensus meeting on cartilage repair of the ankle. Foot Ankle Int 2018;39(1_suppl):28S-34S. https://doi.org/10.1177/107110071878 1098. 
[10] Mittwede PN, Murawski CD, Ackermann J, et al. Revision and salvage management: proceedings of the international consensus meeting on cartilage repair of the ankle. Foot Ankle Int 2018;39(1_suppl):54S-60S. https://doi.org/10.1177/ 1071100718781863.

[11] Reilingh ML, Murawski CD, DiGiovanni CW, et al. Fixation techniques: proceedings of the international consensus meeting on cartilage repair of the ankle. Foot Ankle Int 2018;39(1_suppl):23S-7S. https://doi.org/10.1177/1071100718781096.

[12] Rothrauff BB, Murawski CD, Angthong C, et al. Scaffold-based therapies: proceedings of the international consensus meeting on cartilage repair of the ankle. Foot Ankle Int 2018;39(1_suppl):41S-7S. https://doi.org/10.1177/ 1071100718781864.

[13] Shimozono Y, Brown AJ, Batista JP, et al. Subchondral pathology: proceedings of the international consensus meeting on cartilage repair of the ankle. Foot Ankle Int 2018;39(1_suppl):48S-53S. https://doi.org/10.1177/1071100718781866.

[14] Smyth NA, Murawski CD, Adams Jr SB, et al. Osteochondral Allograft: proceedings of the international consensus meeting on cartilage repair of the ankle. Foot Ankle Int 2018;39(1_suppl):35S-40S. https://doi.org/10.1177/1071100718781097.

[15] van Bergen CJA, Baur OL, Murawski CD, et al. Diagnosis: history, physical examination, imaging, and arthroscopy: proceedings of the international consensus meeting on cartilage repair of the ankle. Foot Ankle Int 2018;39(1_suppl):3S-8S. https://doi.org/10.1177/1071100718779393.

[16] van Dijk PAD, Murawski CD, Hunt KJ, et al. Post-treatment follow-up, imaging, and outcome scores: proceedings of the international consensus meeting on cartilage repair of the ankle. Foot Ankle Int 2018;39(1_suppl):68S-73S. https://doi.org/ $10.1177 / 1071100718781861$.
[17] Linstone HA, Turoff M. Introduction. In: Linstone HA, Turoff M, editors. The Delphi Method: techniques and applications; 2002. p. 1-12.

[18] Ramponi L, Yasui Y, Murawski CD, et al. Lesion size is a predictor of clinical outcomes after bone marrow stimulation for osteochondral lesions of the talus: a systematic review. Am J Sports Med 2017;45(7):1698-705. https://doi.org/ 10.1177/0363546516668292.

[19] Hannon CP, Murawski CD, Fansa AM, Smyth NA, Do H, Kennedy JG. Microfracture for osteochondral lesions of the talus: a systematic review of reporting of outcome data. Am J Sports Med 2013;41(3):689-95. https://doi.org/10.1177/ 0363546512458218.

[20] Shimozono Y, Hurley ET, Yasui Y, Deyer TW, Kennedy JG. The presence and degree of bone marrow edema influence midterm clinical outcomes after microfracture for osteochondral lesions of the talus. Am J Sports Med 2018;46(10):2503-8. https:// doi.org/10.1177/0363546518782701.

[21] Shimozono Y, Yasui Y, Hurley ET, Paugh RA, Deyer TW, Kennedy JG. Concentrated bone marrow aspirate may decrease postoperative cyst occurrence rate in autologous osteochondral transplantation for osteochondral lesions of the talus. Arthroscopy 2019;35(1):99-105. https://doi.org/10.1016/j.arthro.2018. 06.047.

[22] Savage-Elliott I, Smyth NA, Deyer TW, et al. Magnetic resonance imaging evidence of postoperative cyst formation does not appear to affect clinical outcomes after autologous osteochondral transplantation of the talus. Arthroscopy 2016;32(9): 1846-54. https://doi.org/10.1016/j.arthro.2016.04.018.

[23] Wright JG, Swiontkowski MF, Heckman JD. Introducing levels of evidence to the journal. J Bone Joint Surg Am 2003;85(1):1-3. pmid: 12533564. 